\title{
DIRECTLY AND PARAMETRICALLY EXCITED BI-STABLE VIBRATION ENERGY HARVESTER FOR BROADBAND OPERATION
}

\author{
Y. Jia and A.A. Seshia \\ Nanoscience Centre, University of Cambridge, Cambridge, UK
}

\begin{abstract}
Despite many recent advances, the wide-spread adoption of vibrational energy harvesting has been limited by the low levels of generated output power and confined operational frequency band. Recent work by the authors on parametrically excited harvesters has demonstrated over an order of magnitude power improvement. This paper presents an investigation into the simultaneous employment of both direct and parametric resonance, as well as the incorporation of bi-stability, in an attempt to further improve the mechanical-to-electrical energy conversion efficiency by broadening the output power spectrum. Multiple direct and parametric resonant peaks from a multi-degree-of-freedom system were observed and an accumulative $\sim 10 \mathrm{~Hz}$ half-power bandwidth was recorded for the first $40 \mathrm{~Hz}$. Real vibration data was also employed to analysis the rms power response effectiveness of the proposed system.
\end{abstract}

\section{KEYWORDS}

Parametric resonance, bi-stability, vibration, energy harvester, frequency, broadband

\section{INTRODUCTION}

In the past few years, there has been a surge of interest from both the academia and the industry to harness ambient vibration for enabling self-powered wireless sensor networks in structural health monitoring applications. The conventional design approach is to match the natural frequency of an electrically damped resonator with the excitation frequency, in an attempt to activate the fundamental mode of directly excited resonance. However, the reported power output is often insufficient for many practical applications and the operational frequency bandwidth is also often limited. Following a recent paper by the authors [1] that demonstrated over an order of magnitude power enhancement by employing parametric resonance as a means of mechanical amplification, this paper presents an attempt to further improve the mechanical-to-electrical conversion efficiency through broadening the frequency band by simultaneously incorporating both direct and parametric resonant regimes as well as inducing bistability into the base resonator.

\section{BACKGROUND}

Parametric resonance is a particular type of resonant phenomenon that can be induced when at least one of the system parameters oscillates with time with the direction of applied excitation typically orthogonal to the generated displacement. The 1st order or principal parametric resonance can be accessed when the driving frequency is approximately twice the natural frequency and the response frequency is half of the excitation. Any resonator can potentially exhibit both direct and parametric resonance, but is only most responsive to one, depending on the excitation criteria. Therefore, for clarity, this paper shall define a resonator configured to displace parallel to the forced excitation as a primarily direct resonator (DR) and a resonator configured to displace perpendicular to forced excitation as a primarily parametric resonator (PR).

Intrinsically, parametrically excited resonance is associated with higher energy storage than directly excited resonance, as linear damping does not saturate amplitude growth. Although vibrational nonlinearities that are almost always associated with parametric resonance can potentially result in a moderate broadening of the frequency response as compared to the linear directly excited counterparts, the operational band is still narrow. Therefore, when faced with the random and broadband nature of real vibration [2], both direct and parametric resonant regimes are only occasionally activated.

Additionally, the activation of parametric resonance requires stricter boundary conditions and longer transient build up time than direct resonance. This paper proposes an intrinsically multi-frequency complementary harvester that has ready access to multiple direct resonant peaks; and when the boundary conditions become favourable, the more effective parametric resonance can be called upon.

\section{INCORPORATING BI-STABILITY}

\section{Background and motivation}

A major deterrent to the strict boundary conditions of parametric resonance is the presence of a dampingdependent initiation threshold amplitude, which the excitation needs to attain prior to accessing the more "profitable" regions of this resonant phenomenon. The addition of an orthogonal initial clamped-clamped beam (CCB) spring, such as shown in Figure 1a, helps to passively reduce this activation barrier by amplifying the base excitation [1].
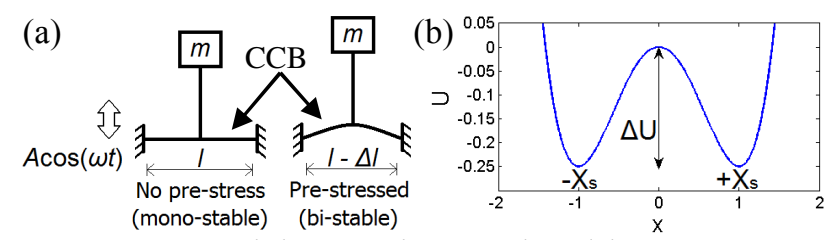

Figure 1: Bi-stability can be introduced by pre-stressing the clamped-clamped beam (CCB) in this previously reported threshold-aided parametric harvester design [1].

The aim is to investigate the potential correlation between bi-stability and the peak power attainable, as well as the initiation threshold of parametric resonance. Bistability can be introduced into this system by reducing the distance between the clamps, thus, pre-stressing the CCB. A symmetric bi-stable system has an unstable equilibrium at zero displacement and two stable equilibria 
positioned on either sides of the origin as shown in Figure $1 \mathrm{~b}$ and defined by the generic equations below.

$$
\begin{aligned}
& m \ddot{x}+c \dot{x}+\dot{U}(x)=F(t) \\
& U(x)=-0.5 k x^{2}+0.25 \mu x^{4}
\end{aligned}
$$

where, $U, x, m, c, F, k$ and $\mu$ are potential energy, displacement, mass, damping, driving force, linear negative spring constant and the Duffing parameter respectively. Equation 3 defines the position of potential intra-wells $\pm x_{s}$ and Equation 4 represents the potential barrier $\Delta U$ (energy required to hop across to the other stable state).

$$
\begin{aligned}
& \pm x_{s}= \pm \sqrt{k / \mu} \\
& \Delta U=k^{2} / 4 \mu
\end{aligned}
$$

Whenever the system hops from one intra-well to another, i.e. the snap-through state for the CCB, a relatively large amount of energy is released that may be electrically harvested [3].

\section{Design, experiment and results}

The design and experimental setup (component volume: $\sim 8.14 \mathrm{~cm}^{3}$ ) in Figure 2 was used to characterise the effects of bi-stability arising from pre-stress. The CCB acts as the initial spring for the subsidiary PR. A piezoelectric ceramic (PZT), by APC International, was employed as the mechanical-to-electrical transducer and was attached to the cantilever using epoxy adhesive.
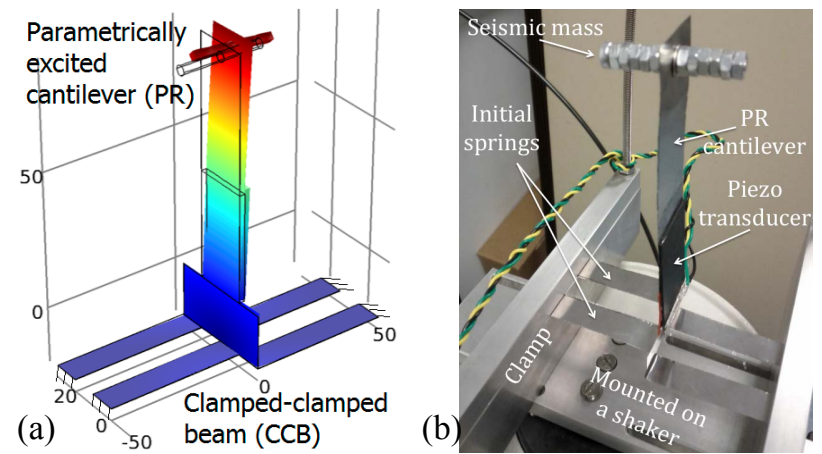

Figure 2: Primarily parametrically excited cantilever beam attached to a clamped-clamped beam.

COMSOL Solid Mechanics simulation revealed the fundamental mode of PR at around $10 \mathrm{~Hz}$ and the fundamental mode of the $\mathrm{CCB}$ at approximately twice that frequency as presented in Figure 3. No parametric resonant modes can be observed within this frequency domain as the underlying physical models are not included in the software package. The small peak right after the cantilever fundamental mode is a lateral mode (sideward motion). Experimentally, the principal parametric resonance of PR was recorded (Figure 4) and the lateral mode was absent due to the negligible resulting transverse strain communicated to the PZT transducer. The measurements were taken using a manual frequency sweep with prolonged single frequency excitation carried out at over a number of discrete frequency steps in order to allow for parametric resonance to build up.

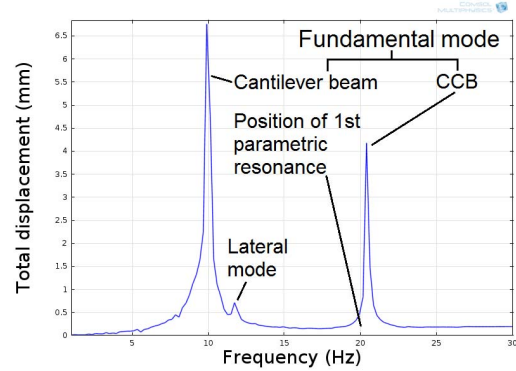

Figure 3: COMSOL Solid Mechanics frequency sweep of Figure 2 a model.

By adjusting the size and position of seismic mass, the natural frequency of the $\mathrm{CCB}$ can be tuned to either match or mismatch the principal parametric resonance of the PR. When frequency matching does take place, autoparametric resonance can be activated. This is a subset of parametric resonance induced by an internal transfer of energy arising from a certain integer ratio relationship in the natural frequencies of the constituting resonating elements. The fundamental mode of CCB and the principal parametric mode of PR do not co-exist and the system alternates between the two modes of resonance. The characteristic identifier of principal parametric resonance is that the excitation frequency is twice that of the observed response.

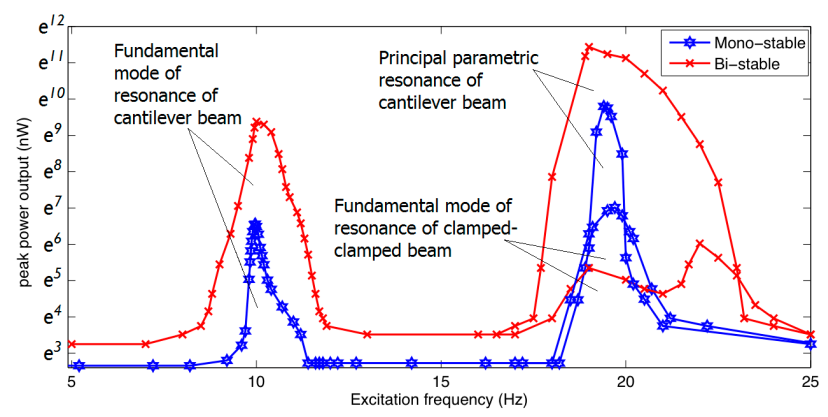

Figure 4: Experimentally measured power spectrum for the design shown in Figure 2 with and without bistability. Parametric resonance can be observed experimentally but lateral mode was not measured by the PZT.

Varying levels of pre-stress was applied to the CCB and a constant trend was observed as shown in Figure 4. With increasing bi-stability, a significant rise in direct and parametric resonant peaks of the PR was experimentally observed. On the other hand, the CCB showed a decrease in quality factor and witnessed the onset of twin resonant peaks around the original natural frequency. This observation agrees with the COMSOL simulation, where the CCB appears to possess a slightly different resonant frequency associated with the different potential intrawells (snap-through state). Table 1 presents peak power results obtained for an excitation input acceleration of $\sim 5$ $\mathrm{ms}^{-2}$, load resistance of $0.38 \mathrm{M} \Omega$ and clamp gaps of 80 $\mathrm{mm}$ (mono-stable), $70 \mathrm{~mm}$ (mildly bi-stable) and $60 \mathrm{~mm}$ (highly bi-stable). 
Table1: Power peaks of various fundamental modes of $P R$ and $C C B$ as well as principal parametric mode of $P R$ at varying levels of bi-stability driven at a constant $\sim 5 \mathrm{~ms}^{-2}$.

\begin{tabular}{|r|c|c|c|}
\hline $\begin{array}{l}\text { Power peaks } \\
(\boldsymbol{\mu W})\end{array}$ & $\begin{array}{c}\text { Mono- } \\
\text { stable }\end{array}$ & $\begin{array}{c}\text { Mildly } \\
\text { bi-stable }\end{array}$ & $\begin{array}{c}\text { Highly } \\
\text { bi-stable }\end{array}$ \\
\hline PR (direct) & 0.70 & 3.20 & 11.8 \\
\hline CCB (direct) & 1.11 & 0.61 & $0.21,0.41$ \\
\hline PR (parametric) & 18.0 & 53.9 & 92.8 \\
\hline
\end{tabular}

Although, once activated, the PR (operated at parametric resonance) performed substantially better with higher bi-stability, the limiting barrier of the initiation threshold required to activate it also increased as follows,

- Mono-stable: $3.60 \mathrm{~ms}^{-2}$

- Mildly bi-stable: $4.05 \mathrm{~ms}^{-2}$

- Highly bi-stable: $4.58 \mathrm{~ms}^{-2}$

This behaviour is a result of lower vibrational response from the stiffer pre-stressed $\mathrm{CCB}$, which is used to amplify the base excitation for PR. Therefore, the effectiveness of the $\mathrm{CCB}$ as a passive aid towards lowering the initiation threshold for parametric resonance is reduced.

\section{DIRECT, PARAMETRIC AND BI-STABLE Design}

Although parametric resonance can offer significantly higher energy conversion efficiency than its directly excited counterparts, its initial activation needs to fulfil a list of critical criteria as follows.

- $\omega=\omega_{\mathrm{n}} / 2$; where $\omega, \omega_{\mathrm{n}}$ and $\mathrm{n}$ are the excitation frequency, natural frequency and an integer denoting the order of parametric resonance respectively.

- Excitation amplitude must overcome the damping dependent initiation threshold amplitude.

- There must be a non-zero initial displacement.

- A prolonged transient build up time must be endured prior to attaining the parametric resonant peak.

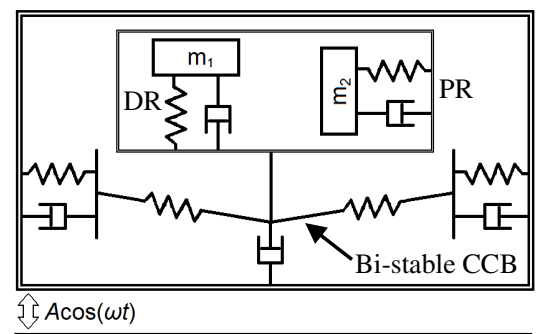

Figure 5: Model view of directly and parametrically excited bi-stable resonator with side springs.

Therefore, employing the more accessible direct resonance alongside parametric resonance helps maximise the response from random vibration input. In the case of bi-stability, the largest energy is released during the snapthrough states. However, crossing the potential barrier requires a large energy input. Additional side springs replacing the anchored clamps of the pre-stressed CCB can help to modulate the height of the potential barrier and increase the probability of snap-through. The overview concept of this directly and parametrically excited bi-stable resonator can be represented in the model diagram shown in Figure 5.

The parameters $m_{1}, m_{2}$ and $A$ denote effective mass of the DR, effective mass of the PR and the amplitude of the external acceleration. From the model diagram, the resonator with displacement parallel to excitation is considered as the primarily directly excited resonator (DR) and vice versa for PR. A design iteration of this, showing both the COMSOL model and the experimental prototype, is presented in Figure 6.

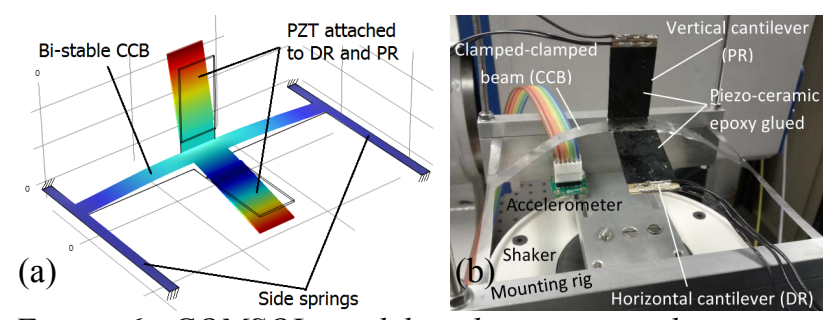

Figure 6: COMSOL model and experimental prototype for the concept presented in Figure 5.. With vertical excitation, the vertical cantilever acts as $P R$ and the horizontal cantilever acts as DR. Both subsidiary resonators rest on a pre-stressed $C C B$. The two side springs aid the modulation of the potential barrier and increase the probability of snap-through.

Figure 7 illustrates the potential barrier modulation in the bi-stable system. As the side springs vibrate, the prestress applied to the $\mathrm{CCB}$ also varies with a time period $\mathrm{T}$. At times $\mathrm{T} / 4$ and 3T/4 the potential barrier is smallest as the vibrating side springs alleviates the pre-stress. Time instants $0 \mathrm{~T}$ and $\mathrm{T} / 2$ correspond to when the side springs return to their respective origins and the potential barrier is unchanged. This increases the probability of crossing over to the neighbouring intra-well. Energy transfer can be maximised if the resonant modes of the side springs are also activated.

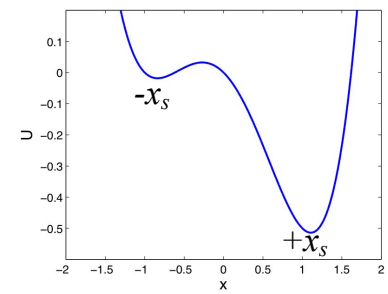

(a) $\mathrm{t}=\mathrm{T} / 4$

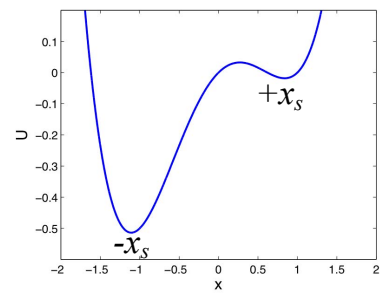

(b) $\mathrm{t}=3 \mathrm{~T} / 4$
Figure 7: Graph showing a representative modulation in the height of the potential barrier for the bi-stable system with side-springs, enabling a higher probability of hopping over to the neighbouring potential intra-well. The parameter $T$ is the time period associated with the modulation.

\section{Results}

Piezo-ceramics are attached to the PR and DR using epoxy adhesive. Unlike the prototype shown in Figure 2, no seismic end mass was attached to the resonators for this characterisation study. Therefore, the PZT strain concentration and absolute power level attainable was significantly lower and larger excitation acceleration was also required to overcome the initiation threshold. 
The experimentally observed power spectrum plotted in Figure 8 shows that both DR and PR are capable of exhibiting direct and parametric resonant peaks, but they are most responsive for the respective type of resonance that they are primarily designed for. No regular and repeatable snap-through motion was recorded for this particular setup due to the stiff tethering of the transducer wires.

The values of the peak power output recorded are presented in Table 2. The applied excitation acceleration was $\sim 30 \mathrm{~ms}^{-2}$ and larger than the initiation threshold required for the onset of parametric resonance for this bistable system. With further increase in excitation, the parametric resonant peaks increase at a much quicker rate than their direct counterparts [1].

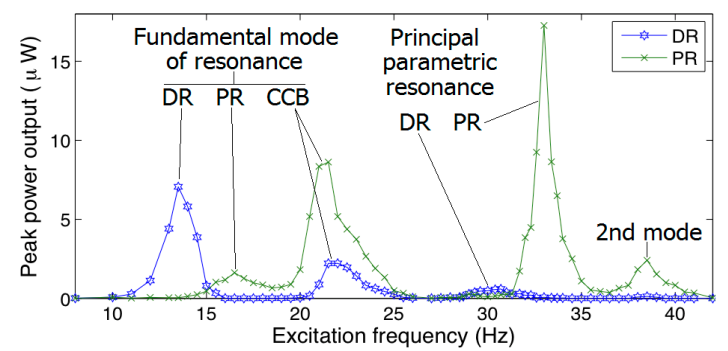

Figure 8: Experimentally measured power spectrum for the design shown in Figure 6 at $\sim 3 \mathrm{~g}$ acceleration.

Table 2: Power peaks of various resonant modes within first $40 \mathrm{~Hz}$ of the directly and parametrically excited bistable harvester driven at $\sim 3 \mathrm{~g}$ acceleration.

\begin{tabular}{|r|c|}
\hline Resonant peak (harvested from) & Peak power $(\mu \mathrm{W})$ \\
\hline DR fundamental mode (DR) & 7.06 \\
\hline PR fundamental mode (PR) & 1.62 \\
\hline CCB fundamental mode (DR) & 2.21 \\
\hline CCB fundamental mode (PR) & 8.62 \\
\hline DR principal parametric (DR) & 0.59 \\
\hline PR principal parametric (PR) & 17.3 \\
\hline PR 2nd direct mode (PR) & 2.41 \\
\hline
\end{tabular}

The fundamental mode of $\mathrm{CCB}$ is transduced from both the DR and PR via the propagated vibration. The second resonant mode of the PR from direct excitation was observed within $40 \mathrm{~Hz}$. However, higher modes of the DR did not surface within this frequency range as predicted by simulation. This could be a result of insufficient excitation for the damping associated with the DR for the higher modes. The PZT transducers employed here were also unresponsive to lateral modes as expected. The half power bands of the recorded peaks accumulated to $\sim 10 \mathrm{~Hz}$ over the measured frequency band. In terms of varying bi-stability, correlations with the amplitude of the peak power and the initiation threshold were in general agreement with the previous section.

\section{Real vibration analysis}

The prototype in Figure $6 \mathrm{~b}$ was subjected to measured vibration data (amplified 12 times) from a railway track and the voltage response from the DR and PR transducers can be seen in Figure 9. Input vibration had random frequency peaks covering a span of nearly 60 $\mathrm{Hz}$ with rapid frequency variations. A FFT analysis of the voltage output confirms a broadband response. As seen from this initial experiment, this multi-frequency harvester is more responsive than a solely direct resonator for broadband real vibration and incorporates the possibility of activating the high conversion efficiency associated with parametric resonance when boundary conditions become favourable.

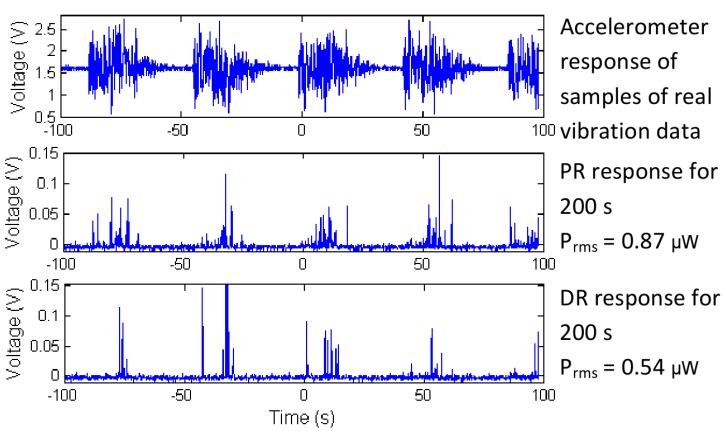

Figure 9: Experimentally recorded voltage response of the prototype shown in Figure $6 b$ to an amplified sample of real vibration data.

\section{CONCLUSION}

This paper builds upon a recent work by the authors on utilising parametric resonance for power response enhancement. The correlation of introducing bi-stability on peak power as well as the initiation threshold amplitude required to activate parametric resonance have been explored. A significant increase in both the fundamental mode peak and principal parametric peak has been recorded with increased bi-stability through a prestressed CCB. A directly and parametrically excited bistable prototype also demonstrated broadband operation by covering approximately a third of accumulated bandwidth between $10 \mathrm{~Hz}$ to $40 \mathrm{~Hz}$. This multi-frequency design readily offers directly excited peaks, while the more effective parametric resonance can also be called upon when boundary conditions become favourable. Future work involves design optimisation to maximise the absolute power response and porting this concept to a volume manufacturing approach. Further investigation will also involve enhancement of potential barrier modulation and methods to lower the initiation threshold for the PR mode of operation.

\section{REFERENCES}

[1] Y. Jia, J. Yan, K. Soga and A.A. Seshia, "Parametrically Excited MEMS Vibration Energy Harvesters", in Digest Tech. Papers PowerMEMS '12, Atlanta, December 2-5, 2012, pp. 215-218.

[2] L. Tang, Y. Yang and C.K. Soh, "Toward Broadband Vibration-based Energy Harvesting", J. of Intell. Mater. Syst., vol. 21, pp. 1867-1897, 2010.

[3] S. Jung and K. Yun, "Energy-harvesting device with mechanical frequency-up conversion mechanism for increased power efficiency and wideband operation", Appl. Phys. Lett., Vol. 96, pp. 111906-1-3, 2010.

\section{CONTACT}

*Y. Jia, Tel.: +44(0)1223 760304; yj252@,cam.ac.uk 